\title{
EFFECT OF HYPERVITAMINOSIS A ON THE FETUS OF SEVERAL SPECIES OF RODENTS, WITH SPECIAL REFERENCE TO MALFORMATION OF THE EXTREMITIES ${ }^{11}$
}

\author{
MASAHIRO MIZUTANI, TOSHIO IHARA AND KYO KAZIWARA \\ Biological Research Laboratories, Research and Development Division, \\ Takeda Chemical Industries, Ltd., Osaka
}

Received September 22, 1965

Since a teratogenic effect of excess viamin $\mathrm{A}$ on the rat fetus was first reported by Cohlan in 1953, many investigators have confirmed and expanded his results (Giroud and Martinet 1955, 1956, 1958; Millen and Woollam 1957, 1963; Baba and Araki 1959; Deuschle et al. 1959; Kalter 1960; Kalter and Warkany 1961; Woollam and Millen 1961; Murakami et al. 1963, 1965; Takekoshi 1964; Ohzu and Shoji 1965). They have described the occurrence of various types of anomalies such as gross malformations which are associated with the central nervous system, a certain combination of defects called the anomalous oculodentofacial pattern by Deuschle et al. (1959), cleft palate, micromelia and other malformations of the limb, digital anomalies, anal atresia, and malformations of vertebrae and ribs as well as some visceral changes mainly in rodents. Parallel experiments by the same investigator using more than two species of laboratory animals however, are few except for a brief report by Giroud and Martinet (1958). It is difficult to discuss difference in teratogenic action of hypervitaminosis $\mathrm{A}$ among species on the basis of the results previously published by different investigators, because of differences in the strain of animals, time and route of administration and the form and dosage of vitamin $\mathrm{A}$ as well as in the object of observation in each study.

To discuss difference in the teratogenic effect of an extrinsic agent among species, not only constancy of the factors which may influence upon the teratogenic action of the agent (Wilson 1959, Fraser 1963) but also parallel experiments done by the same investigator under the same technical level during the same period is required. From this viewpoint parallel experiments on hypervitaminosis A were carried out in mice, rats and rabbits in the present investigation. Numerous malformations of the same type previously described in the literature were found. In carrying out the present study, special attention was paid to differences in the occurrence of limb and digital malformations among species and the following relationships were examined. 1) The relationship between time of administration and the frequency of fetal deaths

1) This paper is dedicated to Professor Sajiro Makino (Zoological Institute, Faculty of Science, Hokkaido Uuiversity, Sapporo, Japan) in honor of his 60th birthday, June 21, 1966 from the senior author M. M. 
including embryonic losses as well as of limb and digital malformations. 2) The relationship between dose and incidence of limb and digital malformations.

\section{MATERIALS AND METHODS}

The animals used in the experiments were closed colonies of CF-1 mice* and Sprague Dawley rats* and white rabbits** which were $F_{1}$ of paired animals (random mating) selected from several inbred lines (7-8th generation). Each species was kept in an animal room with air-conditioning under a temperature at $24 \pm 1^{\circ} \mathrm{C}$ and a humidity of $55 \pm 10 \%$. In mice and rats a virgin female in estrous stage was kept overnight in a cage with a male, and if a vaginal plug (mouse, rat) or spermatozoa (rat) was found the next morning, she was considered to be in the first day of pregnancy. In rabbits, the day of confirmed copulation was regarded as the zero day of pregnancy.

The experiments were divided into three sections. In the first section, the equivalent ages of developing embryos in mice, rats and rabbits were determined by grossly comparing morphogenetic patterns such as number of somites, condition of the neural tube, eyes, maxillary processes, limb buds, and hand or foot plates. Observation of the developmental stage was limited to the susceptible period of embryos to the teratogens. In the second section (stage-reponse experiments), mice were given a single, intraperitoneal injection of water-miscible vitamin A palmitate in a dose of $600,000 \mathrm{IU} / \mathrm{kg}$ from the 8 th to the 13 th day of pregnancy, rats were given a dose of $500,000 \mathrm{IU} / \mathrm{kg}$ from the 10 th to the 15 th day, and rabbits were administered $300,000 \mathrm{IU} / \mathrm{kg}$ from the 8 th to the 16th day. The dosages used were determined by the subacute toxicity test for adult females and embryonic toxicity test for embryonal LD $_{100}$ in females treated on the 8th day of pregnancy in each species. The time of administration was based on both the references previously reported and the observation of equivalent embryonic ages in the three species employed. In the third part (doseresponse experiments), various dose levels of vitamin A $(50,000-250,000 \mathrm{IU} / \mathrm{kg})$ were administered in a single dose by the intraperitoneal route on the 10th day of pregnancy in mice, on the 11th day in rats and on the 10th day in rabbits. In this case, the time of administration was based on the stage-response experiments. Through the period of these experiments, a large number of pregnant animals in each species used were employed as the untreated control.

The pregnant animals were delivered by Saesarean section on the 19th day of pregnancy in mice, on the 21st day in rats (one day before expected date of delivary) and on the 28th day in rabbits (three days prior to date of delivary). The fetuses were weighed and then examined for gross malformations under a binocular. The

* Supplied by Section of Experimental Animals, Department of Bacteriology. Hikari Plant, Takeda Chemical Industries, Ltd.

** Supplied by Farm in Tokyo Plant, Takeda Chemical Industries, Ltd. 
fetuses were later examined for skeletal malformations under the same binocular after being processed by the modified Schulze-Dawson method (Dawson 1926).

\section{RESULTS}

\section{Equivalent ages of development in the three species}

Embryonic age at the time of teratogenic action is accepted as an important determinant. Equivalent ages in the rat and rabbit embryos for each successive mean age of the mouse embryos from the 8th to the 13th day of pregnancy were grossly determined by comparing several morphogenetic patterns such as number of somites, closure
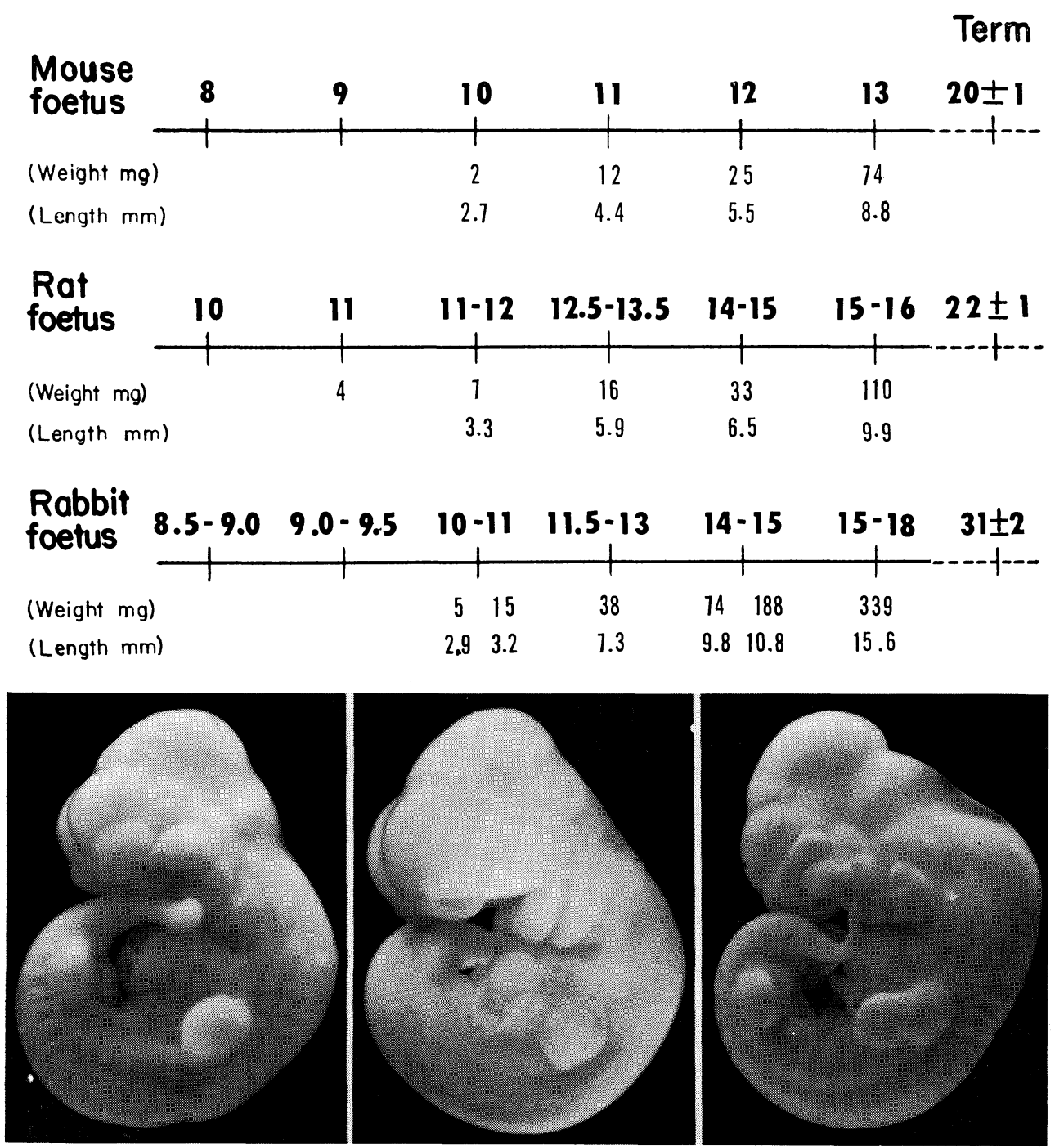

Text-fig. 1. Equivalent ages in mouse (11th day), rat (13th day), and rabbit (13th day) embryos. 
of neural tube, and developmental state of the eyes, maxillary processes, limb buds and hand or foot plates. The results are summarized in Text-figure 1 . The 8th, 10th and 13th day of pregnancy in mice corresponded to the 9th-10th, 11th-12th and 15th16 th day in rats, and to the 8 th -9 th, 10 th- 11 th and 15 th-18th day in rabbits, respectively. (Details will be reported elswhere)

\section{Stage-response experiments}

The number of animals treated, total number of fetuses examined, the frequency of fetal deaths including embryonic losses, the incidence of malformations and other pertinent information are presented in Tables 1-3.

Table 1. Pregnancy status of mice treated with hypervitaminosis-A $(600,000 \mathrm{IU} / \mathrm{kg})$ at different stage of pregnancy

\begin{tabular}{|c|c|c|c|c|c|c|c|}
\hline Observation points & $\begin{array}{l}8 \text { th } \\
\text { day }\end{array}$ & $\begin{array}{l}9 \text { th } \\
\text { day }\end{array}$ & $\begin{array}{l}\text { 10th } \\
\text { day }\end{array}$ & $\begin{array}{l}\text { 11th } \\
\text { day }\end{array}$ & $\begin{array}{l}12 \text { th } \\
\text { day }\end{array}$ & $\begin{array}{l}13 \text { th } \\
\text { day }\end{array}$ & CONT. \\
\hline No. of mice treated & 5 & 15 & 12 & 20 & 21 & 16 & 71 \\
\hline Total number of fetuses & 48 & 127 & 102 & 171 & 189 & 141 & 638 \\
\hline Fetal deaths & $\begin{array}{r}47 \\
(97.9) \\
\end{array}$ & $\begin{array}{c}32 \\
(25.2) \\
\end{array}$ & $\begin{array}{c}20 \\
(19.6)\end{array}$ & $\begin{array}{c}20 \\
(11.7) \\
\end{array}$ & $\begin{array}{c}17 \\
(9.0)\end{array}$ & $\begin{array}{c}9 \\
(6.4)\end{array}$ & $\begin{array}{c}78 \\
(12.2)\end{array}$ \\
\hline Fetuses examined & 1 & $\begin{array}{l}95 \\
85\end{array}$ & $\begin{array}{l}82 \\
79\end{array}$ & $\begin{array}{l}151 \\
149\end{array}$ & $\begin{array}{l}172 \\
170\end{array}$ & $\begin{array}{l}132 \\
109\end{array}$ & $\begin{array}{l}560 \\
548\end{array}$ \\
\hline $\begin{array}{l}\text { No. of fetuses with gross } \\
\text { malformations }\end{array}$ & "1" & $\begin{array}{c}81 \\
(85.3)\end{array}$ & $\begin{array}{c}79 \\
(96.3)\end{array}$ & $\begin{array}{c}144 \\
(95.4)\end{array}$ & $\begin{array}{c}42 \\
(24.4) \\
\end{array}$ & $\begin{array}{c}6 \\
(4.5)\end{array}$ & $\begin{array}{l}16 \\
(2.9)\end{array}$ \\
\hline Limb malformations & "0" & $\begin{array}{c}41 \\
(43.2)\end{array}$ & $\begin{array}{c}74 \\
(90.2)\end{array}$ & $\begin{array}{l}138 \\
(91.4)\end{array}$ & $\begin{array}{c}9 \\
(5.2)\end{array}$ & 0 & 0 \\
\hline Digital malformations & $" 0 "$ & $\begin{array}{c}31 \\
(32.6)\end{array}$ & $\begin{array}{c}68 \\
(82.9)\end{array}$ & $\begin{array}{l}142 \\
(94.0)\end{array}$ & $\begin{array}{c}30 \\
(17.4)\end{array}$ & 0 & 0 \\
\hline $\begin{array}{l}\text { No. of fetuses with bone } \\
\text { abnormalities }\end{array}$ & $" 1 "$ & $\begin{array}{c}81 \\
(95.3)\end{array}$ & $\begin{array}{c}79 \\
(100.0)\end{array}$ & $\begin{array}{l}145 \\
(97.3)\end{array}$ & $\begin{array}{c}48 \\
(32.2)\end{array}$ & $\begin{array}{c}12 \\
(11.0)\end{array}$ & $\begin{array}{c}23 \\
(4.2) \\
\end{array}$ \\
\hline $\begin{array}{l}\text { Abnormalities in the long bones } \\
\text { of the extrimities }\end{array}$ & $" 0 "$ & $\begin{array}{c}76 \\
(89.4)\end{array}$ & $\begin{array}{c}70 \\
(88.6)\end{array}$ & $\begin{array}{l}140 \\
(93.9)\end{array}$ & $\begin{array}{l}12 \\
(7.1)\end{array}$ & 0 & $\begin{array}{c}9 \\
(1.6)\end{array}$ \\
\hline
\end{tabular}

The frequency of fetal deaths, including resorptions and placental remnants, in each species was striking in the earliest stage treated, while, the frequency gradually decreased as the embryos grew older. The incidence of gross malformations was also remarkable in all species used, when the animals were treated at earlier stages such as the 9th-11th day of pregnancy in mice, 10th-13th day in rats and 9th-11th day in rabbits. The incidence of malformations due to vitamin A injected on the 8 th day in mice and rabbits was not clear because of extremely high embryonic mortality. Characteristic malformations were manifested according to the stage at which the agent was administered. Oculofacial syndrome (certain combination of defects such as microcephalia and malformation of the eyes, jaw, mouth and auricle) and malformation of the extremities were unique anomalies. Details on the oculofacial syndrome will be reported elswhere (Ihara et al. 1966). The most prominent type among malformation of the extremities was micromelia such as is sometimes called "phocomelia". Micromelia was produced in those treated on the 9th-12th day of pregnancy in mice 
Table 2. Pregnancy status of rats treated with hypervitaminosis-A $(500,000 \mathrm{IU} / \mathrm{kg})$ at different stage of pregnancy

\begin{tabular}{|c|c|c|c|c|c|c|c|}
\hline Observation points & $\begin{array}{l}\text { 10th } \\
\text { day }\end{array}$ & $\begin{array}{l}\text { 11th } \\
\text { day }\end{array}$ & $\begin{array}{l}12 \text { th } \\
\text { day }\end{array}$ & $\begin{array}{l}\text { 13th } \\
\text { day }\end{array}$ & $\begin{array}{l}\text { 14th } \\
\text { day }\end{array}$ & $\begin{array}{l}15 \text { th } \\
\text { day }\end{array}$ & CONT. \\
\hline No. of rats treated & 21 & 22 & 31 & 26 & 21 & 20 & 126 \\
\hline Total number of fetuses & 216 & 205 & 327 & 260 & 193 & 223 & 1244 \\
\hline Fetal deaths & $\begin{array}{c}36 \\
(16.6)\end{array}$ & $\begin{array}{c}37 \\
(18.0)\end{array}$ & $\begin{array}{l}21 \\
(6.4)\end{array}$ & $\begin{array}{l}10 \\
(4.0)\end{array}$ & $\begin{array}{l}11 \\
(5.7)\end{array}$ & $\begin{array}{c}9 \\
(4.0)\end{array}$ & $\begin{array}{l}48 \\
(3.9)\end{array}$ \\
\hline Fetuses examined & $\begin{array}{l}180 \\
160\end{array}$ & $\begin{array}{l}168 \\
168\end{array}$ & $\begin{array}{l}306 \\
299\end{array}$ & $\begin{array}{l}250 \\
235\end{array}$ & $\begin{array}{l}182 \\
168\end{array}$ & $\begin{array}{l}214 \\
214\end{array}$ & $\begin{array}{l}1196 \\
1167\end{array}$ \\
\hline $\begin{array}{l}\text { No. of fetuses with gross } \\
\text { malformations }\end{array}$ & $\begin{array}{l}158 \\
(87.8)\end{array}$ & $\begin{array}{l}126 \\
(75.0)\end{array}$ & $\begin{array}{l}252 \\
(82.4)\end{array}$ & $\begin{array}{l}178 \\
(71.2)\end{array}$ & $\begin{array}{c}52 \\
(28.6)\end{array}$ & $\begin{array}{c}1 \\
(0.5)\end{array}$ & 0 \\
\hline Limb malformations & $\begin{array}{c}43 \\
(23.9)\end{array}$ & $\begin{array}{c}92 \\
(54.8)\end{array}$ & $\begin{array}{l}150 \\
(49.0)\end{array}$ & $\begin{array}{c}43 \\
(17.2)\end{array}$ & 0 & 0 & 0 \\
\hline Digital malformations & $\begin{array}{l}102 \\
(56.7)\end{array}$ & $\begin{array}{l}124 \\
(73.8)\end{array}$ & $\begin{array}{c}240 \\
(78.4)\end{array}$ & $\begin{array}{c}138 \\
(55.2)\end{array}$ & $\begin{array}{c}6 \\
(3.3)\end{array}$ & 0 & 0 \\
\hline $\begin{array}{l}\text { No. of fetuses with bone } \\
\text { abnormalities }\end{array}$ & $\begin{array}{l}143 \\
(89.4)\end{array}$ & $\begin{array}{l}129 \\
(76.8)\end{array}$ & $\begin{array}{l}225 \\
(75.3)\end{array}$ & $\begin{array}{c}97 \\
(41.3)\end{array}$ & $\begin{array}{c}66 \\
(39.3)\end{array}$ & $\begin{array}{l}11 \\
(5.1)\end{array}$ & $\begin{array}{l}17 \\
(1.5)\end{array}$ \\
\hline $\begin{array}{l}\text { Abnormalities in the long bones } \\
\text { of the extrimities }\end{array}$ & $\begin{array}{l}128 \\
(80.0)\end{array}$ & $\begin{array}{l}127 \\
(75.6)\end{array}$ & $\begin{array}{l}221 \\
(73.9)\end{array}$ & $\begin{array}{c}94 \\
(40.0)\end{array}$ & $\begin{array}{c}52 \\
(30.9)\end{array}$ & 0 & 0 \\
\hline
\end{tabular}

Table 3. Pregnancy status of rabbits treated with hypervitaminosis-A $(300,000 \mathrm{IU} / \mathrm{kg})$ at different stage of pregnancy

\begin{tabular}{|c|c|c|c|c|c|c|c|c|c|c|}
\hline 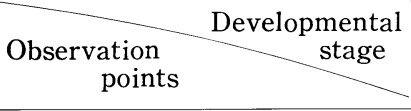 & $\begin{array}{l}8 \text { th } \\
\text { day }\end{array}$ & $\begin{array}{l}9 \text { th } \\
\text { day }\end{array}$ & $\begin{array}{l}\text { 10th } \\
\text { day }\end{array}$ & $\begin{array}{l}11 \text { th } \\
\text { day }\end{array}$ & $\begin{array}{l}\text { 12th } \\
\text { day }\end{array}$ & $\begin{array}{l}\text { 13th } \\
\text { day }\end{array}$ & $\begin{array}{l}14 \text { th } \\
\text { day }\end{array}$ & $\begin{array}{l}\text { 15th } \\
\text { day }\end{array}$ & $\begin{array}{l}16 \text { th } \\
\text { day }\end{array}$ & $\stackrel{\leftrightarrow}{\stackrel{\leftrightarrow}{Z}}$ \\
\hline No. of rabbits treated & 3 & 6 & 5 & 5 & 5 & 5 & 5 & 4 & 4 & 4 \\
\hline Total number of fetuses & 29 & 51 & 42 & 39 & 59 & 53 & 38 & 36 & 31 & 31 \\
\hline Fetal deaths & $\begin{array}{c}29 \\
(100.0)\end{array}$ & $\begin{array}{c}43 \\
(84.3) \\
\end{array}$ & $\begin{array}{c}24 \\
(57.2)\end{array}$ & $\begin{array}{c}15 \\
(38.5)\end{array}$ & $\begin{array}{c}14 \\
(23.6)\end{array}$ & $\begin{array}{c}12 \\
(22.6)\end{array}$ & $\begin{array}{c}1 \\
(2.6) \\
\end{array}$ & 0 & $\begin{array}{c}6 \\
(19.3) \\
\end{array}$ & $\begin{array}{c}1 \\
(3.2)\end{array}$ \\
\hline $\begin{array}{ll}\text { Fetuses examined } & \text { (gross) } \\
\text { (bone) }\end{array}$ & $\begin{array}{l}0 \\
0\end{array}$ & $\begin{array}{l}8 \\
7 \\
\end{array}$ & $\begin{array}{l}18 \\
17 \\
\end{array}$ & $\begin{array}{l}24 \\
20 \\
\end{array}$ & $\begin{array}{l}45 \\
43 \\
\end{array}$ & $\begin{array}{l}41 \\
37 \\
\end{array}$ & $\begin{array}{l}37 \\
30\end{array}$ & $\begin{array}{l}36 \\
36 \\
\end{array}$ & $\begin{array}{l}25 \\
24 \\
\end{array}$ & $\begin{array}{l}30 \\
30\end{array}$ \\
\hline $\begin{array}{l}\text { No. of fetuses with gross } \\
\text { malformations }\end{array}$ & & $\begin{array}{c}4 \\
(50.0) \\
\end{array}$ & $\begin{array}{c}18 \\
(100.0) \\
\end{array}$ & $\begin{array}{c}19 \\
(79.2)\end{array}$ & $\begin{array}{c}26 \\
(57.8) \\
\end{array}$ & $\begin{array}{c}10 \\
(24.4) \\
\end{array}$ & 0 & 0 & $\begin{array}{c}1 \\
(4.0)\end{array}$ & 0 \\
\hline Limb malformations $(\%)$ & & 0 & 0 & 0 & 0 & 0 & 0 & 0 & 0 & 0 \\
\hline Digital malformations $(\%)$ & & 0 & $\begin{array}{c}5 \\
(27.8) \\
\end{array}$ & $\begin{array}{c}3 \\
(12.5) \\
\end{array}$ & $\begin{array}{c}12 \\
(26.7) \\
\end{array}$ & $\begin{array}{c}7 \\
(17.1) \\
\end{array}$ & 0 & 0 & 0 & 0 \\
\hline $\begin{array}{l}\text { No. of fetuses with bone } \\
\text { abnormalities }\end{array}$ & & $\begin{array}{c}2 \\
(28.6) \\
\end{array}$ & $\begin{array}{c}17 \\
(100.0) \\
\end{array}$ & $\begin{array}{c}20 \\
(100.0)\end{array}$ & $\begin{array}{c}32 \\
(74.4)\end{array}$ & $\begin{array}{c}23 \\
(62.2) \\
\end{array}$ & $\begin{array}{c}9 \\
(30.0) \\
\end{array}$ & $\begin{array}{c}4 \\
(11.1) \\
\end{array}$ & $\begin{array}{c}5 \\
(20.8) \\
\end{array}$ & $\begin{array}{c}1 \\
(3.3) \\
\end{array}$ \\
\hline $\begin{array}{l}\text { Abnormalities in the long } \\
\text { bones of the extrimities }(\%)\end{array}$ & & $\begin{array}{c}2 \\
(28.6) \\
\end{array}$ & $\begin{array}{c}8 \\
(47.1) \\
\end{array}$ & $\begin{array}{c}9 \\
(45.0) \\
\end{array}$ & $\begin{array}{c}5 \\
(11.7)\end{array}$ & 0 & 0 & $\begin{array}{c}3 \\
(8.3) \\
\end{array}$ & 0 & 0 \\
\hline
\end{tabular}

and on the 10th-13th day in rats, while no such anomaly was manifested in rabbits except for a minor defect in the long bones of the extremities (Figs. 1-3). The incidene of micromelia in each equivalent age was always higher in mice than in rats and also higher in the forelimb than in the hindlimb except in a group of rats treated on the 13th day. The critical stage for the production of micromelia was the 10th-11th day in mice and the 11th-12th day in rats. The developmental stage at which the frequency was the highest was approximately one day later for the hindlimb than for the forelimb, that is, the critical stage in the forelimb was the 10th- 
11th day in mice and the 11th day in rats, while in the hindlimb, the critical stage was the 11th day in mice and the 11th-12th day in rats.
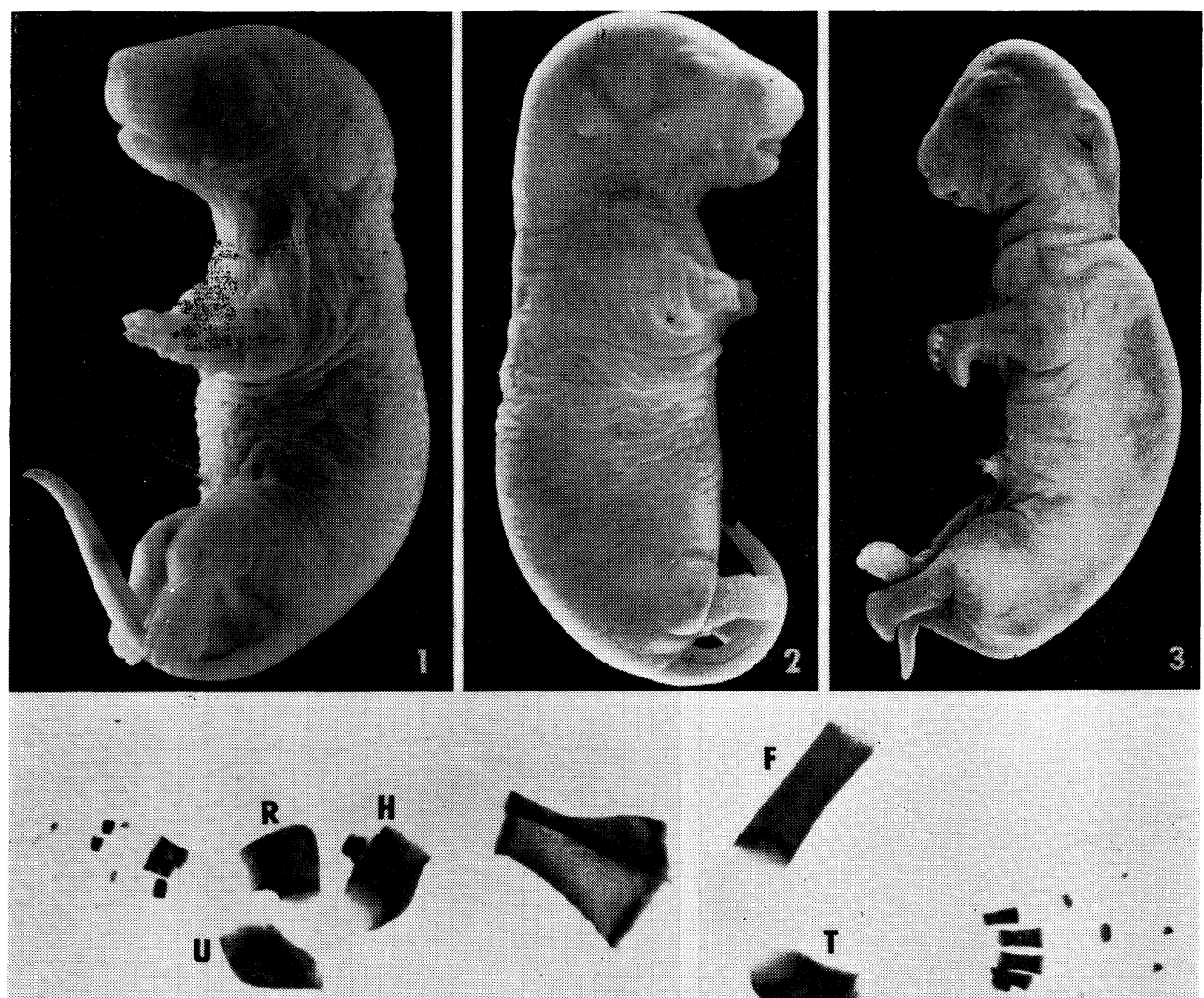

4

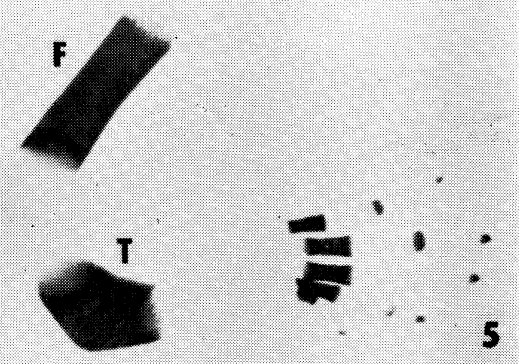

Fig. 1. Offspring of a mouse with micromelia in the forelimb and club foot.

Fig. 2. Offspring of a rat with micromelia in the fore- and hindlimb.

Fig. 3. Offspring of a rabbit with the abnormal extremities.

Fig. 4. A case of micromelia in the forelimb in an alizarin red $\mathrm{S}$ stained mouse specimen. $\mathrm{H}$ : humerus $\mathrm{R}$ : radius $\mathrm{U}$ : ulna

Fig. 5. A case of club foot associated with micromelia in an alizarin red $\mathrm{S}$ stained mouse specimen. Fibula is defficient. F: femur $\mathrm{T}$ : tibia

The susceptible period for the production of digital malformations was the 9th13th day of pregnancy in mice, the 10th-14th day in rats and the 10th-13th day in rabbits. The incidence of digital malformations in each equivalent age was the highest in mice, followed in order by the rat and the rabbit. In mice and rats, ectrodactylia, brachydactylia and syndactylia were maifested, while in rabbits, brachydactylia was absent (Table 4). Ectrodactylia was often associated with micromelia in mice and rats.

In the skeletal system of micromelia, the long bones of the extremities were 
Table. 4 Classification and incidence of limb and digital malformations in the three species treated with hypervitaminosis $\mathrm{A}$ at different stages of pregnancy (For details, see text)

\begin{tabular}{|c|c|c|c|c|c|c|c|c|c|c|c|}
\hline & $\begin{array}{l}\begin{array}{r}\text { Developmental } \\
\text { stage (Day) }\end{array} \\
\text { Malfor- } \\
\text { mations (\%) }\end{array}$ & 8 & 9 & 10 & 11 & 12 & 13 & 14 & 15 & 16 & Control \\
\hline \multirow{9}{*}{. } & Micromelia & 0 & 17 & 69 & 116 & 2 & 0 & - & - & - & 0 \\
\hline & Ectrodactylia & 0 & $\begin{array}{l}(17.0) \\
26\end{array}$ & $\begin{array}{l}(04.1) \\
60\end{array}$ & $\begin{array}{l}(76.8) \\
112\end{array}$ & $\begin{array}{l}(1.2) \\
6\end{array}$ & 0 & - & - & - & 0 \\
\hline & Brachydactylia & 0 & $\begin{array}{l}(27.4) \\
1\end{array}$ & $\begin{array}{l}(73.2) \\
14\end{array}$ & $\begin{array}{l}(74.2) \\
25\end{array}$ & $\begin{array}{c}(3.5) \\
0\end{array}$ & 0 & - & - & - & 0 \\
\hline & Syndactylia & 0 & $\begin{array}{l}(1.1) \\
0\end{array}$ & $\begin{array}{l}(17.1) \\
2\end{array}$ & $\begin{array}{c}(10.5) \\
1\end{array}$ & 0 & 0 & - & - & - & 0 \\
\hline & Micromelia & 0 & 0 & 20 & 93 & 0 & 0 & - & - & - & 0 \\
\hline & Ectrodactylia & 0 & 10 & $\begin{array}{l}(24.4) \\
10\end{array}$ & $\begin{array}{l}(61.6) \\
76\end{array}$ & 8 & 0 & - & - & - & 0 \\
\hline & Brachydactylia & 0 & (10.5) & $(12.2)$ & $(50.4)$ & $(4.7)$ & 0 & - & - & - & 0 \\
\hline & Cundlotuli: & 0 & 4 & $(1.2)$ & $(4.0)$ & $(1.2)$ & 0 & & & & 0 \\
\hline & Syndactyna & 0 & $\begin{array}{l}4 \\
(4.2)\end{array}$ & $(12.2)$ & $\begin{array}{l}35 \\
(23.5)\end{array}$ & $(7.7)$ & & & & & 0 \\
\hline \multirow{9}{*}{ है } & Micromelia & - & - & $\begin{array}{c}42 \\
(23,3)\end{array}$ & 93 & 147 & 28 & 0 & 0 & - & 0 \\
\hline & Ectrodactylia & - & - & 63 & 122 & 144 & 23 & 1 & 0 & - & 0 \\
\hline & Brachydactylia & - & - & 64 & $\begin{array}{l}(73.9) \\
25\end{array}$ & $\begin{array}{l}(40.2) \\
135\end{array}$ & 111 & $\begin{array}{l}(0.5) \\
1\end{array}$ & 0 & - & 0 \\
\hline & Syndactylia & - & - & $\begin{array}{l}(30.0) \\
11\end{array}$ & $\begin{array}{l}(15.2) \\
8\end{array}$ & $\begin{array}{c}(37.7) \\
4\end{array}$ & $\begin{array}{c}(44.4) \\
0\end{array}$ & $\begin{array}{l}(0.5) \\
0\end{array}$ & 0 & - & 0 \\
\hline & Micromelia & - & - & 1 & 55 & 121 & 39 & 0 & 0 & - & 0 \\
\hline & $\Gamma$ & & & $(0.6)$ & (33.3) & $(33.8)$ & $(15.6)$ & 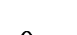 & 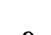 & & \\
\hline & Ectrodactylia & - & - & 400 & 101 & 152 & 53 & 0 & 0 & - & 0 \\
\hline & Brachydactylia & - & - & 11 & 8 & 45 & 58 & 5 & 0 & - & 0 \\
\hline & Syndactylia & - & - & $\begin{array}{l}2 \\
(1.1)\end{array}$ & $\begin{array}{l}4 \\
(2.4)\end{array}$ & $\begin{array}{l}3 \\
(0.8)\end{array}$ & 0 & 0 & 0 & - & 0 \\
\hline \multirow{2}{*}{ 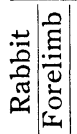 } & Ectrodactylia & 0 & 0 & 3 & 2 & 11 & 7 & 0 & 0 & 0 & 0 \\
\hline & Syndactylia & 0 & 0 & $\begin{array}{c}1 \\
(5.6)\end{array}$ & $\begin{array}{l}1 \\
1 \\
(4.2)\end{array}$ & 0 & 0 & 0 & 0 & 0 & 0 \\
\hline
\end{tabular}

complicated with deficiency, shortening, bending, thickening or a combination (Figs. 4-5). Deficiency of the radius and tibia was rare in contrast to the frequent absence of the ulna and fibula. In the extensively defective case, the humerus, femur, ulna and fibula were absent and radius as well as tibia were usually deformed in a conic mass. In rabbits, slight thickening of the radius and ulna was the only deformity observed in the long bones of the extremities.

\section{Dose-response experiments}

On the basis of the above finding, experiments were undertaken in order to obtain information on the dose-response relationships of limb and digital malformations in mice, rats and rabbits. The number of animals, total number of fetuses examined, the frequency of limb and digital malformations and other pertinent informations are 
Table. 5 Pregnancy status of mice treated with various doses of hypervitaminosis-A $(50,000 \sim 250,000 \mathrm{IU} / \mathrm{kg})$ on the 10 th day of pregnancy

\begin{tabular}{|c|c|c|c|c|c|c|c|c|c|c|}
\hline $\operatorname{c}_{\begin{array}{c}\text { Observation } \\
\text { points }\end{array}}^{\mathrm{Dcse}}\left(\times 10^{4} \mathrm{IU} / \mathrm{kg}\right)$ & CONT. & 5.0 & 7.5 & 10.0 & 12.5 & 15.0 & 17.5 & 20.0 & 22.5 & 25.0 \\
\hline No. of mice treated & 71 & 10 & 10 & 18 & 14 & 10 & 11 & 14 & 12 & 12 \\
\hline Total number of fetuses & 638 & 109 & 84 & 159 & 135 & 97 & 101 & 122 & 118 & 79 \\
\hline Fetal deaths & $\begin{array}{c}78 \\
(12.2)\end{array}$ & $\begin{array}{c}10 \\
(9.2)\end{array}$ & $\begin{array}{c}6 \\
(7.1)\end{array}$ & $\begin{array}{c}10 \\
(6.2)\end{array}$ & $\begin{array}{c}17 \\
(12.6)\end{array}$ & $\begin{array}{c}14 \\
(14.4)\end{array}$ & $\begin{array}{c}8 \\
(8.0)\end{array}$ & $\begin{array}{c}21 \\
(17.2)\end{array}$ & $\begin{array}{c}22 \\
(19.1)\end{array}$ & $\begin{array}{c}8 \\
(10.2)\end{array}$ \\
\hline Fetuses examined $\quad \begin{array}{c}\text { (gross) } \\
\text { (bone) }\end{array}$ & & $\begin{array}{l}99 \\
99\end{array}$ & $\begin{array}{l}78 \\
78\end{array}$ & $\begin{array}{l}149 \\
149\end{array}$ & $\begin{array}{l}118 \\
118\end{array}$ & $\begin{array}{l}83 \\
83 \\
\end{array}$ & $\begin{array}{l}93 \\
92\end{array}$ & $\begin{array}{l}101 \\
101\end{array}$ & 96 & $\begin{array}{l}71 \\
69\end{array}$ \\
\hline $\begin{array}{l}\text { No. of fetuses with gross } \\
\text { malformations }\end{array}$ & $\begin{array}{l}16 \\
(2.9)\end{array}$ & $\begin{array}{c}4 \\
(4.0)\end{array}$ & 0 & $\begin{array}{l}14 \\
(9.4)\end{array}$ & $\begin{array}{c}24 \\
(12.0)\end{array}$ & $\begin{array}{c}35 \\
(42.2)\end{array}$ & $\begin{array}{c}58 \\
(62.4)\end{array}$ & $\begin{array}{c}68 \\
(67.3)\end{array}$ & $\begin{array}{c}74 \\
(77.1)\end{array}$ & $\begin{array}{c}58 \\
(81.7)\end{array}$ \\
\hline Limb malformations $(\%)$ & 0 & $\begin{array}{c}2 \\
(2.0) \\
\end{array}$ & 0 & $\begin{array}{l}10 \\
(6.7)\end{array}$ & $\begin{array}{c}24 \\
(20.3) \\
\end{array}$ & $\begin{array}{c}27 \\
(32.5) \\
\end{array}$ & $\begin{array}{c}54 \\
(58.1) \\
\end{array}$ & $\begin{array}{c}58 \\
(57.4)\end{array}$ & $\begin{array}{c}64 \\
(66.6) \\
\end{array}$ & $\begin{array}{c}54 \\
(76.1)\end{array}$ \\
\hline Digital malformations $(\%)$ & 0 & 0 & 0 & $\begin{array}{c}2 \\
(1.3)\end{array}$ & 0 & $\begin{array}{c}14 \\
(16.8)\end{array}$ & $\begin{array}{c}32 \\
(34.4)\end{array}$ & $\begin{array}{c}37 \\
(36.6)\end{array}$ & $\begin{array}{c}34 \\
(35.4)\end{array}$ & $\begin{array}{c}47 \\
(66.2)\end{array}$ \\
\hline $\begin{array}{l}\text { No. of fetuses with bone } \\
\text { abnormalities }\end{array}$ & $\begin{array}{c}23 \\
(4.2) \\
\end{array}$ & $\begin{array}{c}18 \\
(18.2)\end{array}$ & $\begin{array}{c}7 \\
(9.0) \\
\end{array}$ & $\begin{array}{c}37 \\
(24.8) \\
\end{array}$ & $\begin{array}{c}55 \\
(46.6) \\
\end{array}$ & $\begin{array}{c}59 \\
(71.1)\end{array}$ & $\begin{array}{c}87 \\
(94.6) \\
\end{array}$ & $\begin{array}{l}101 \\
(100.0)\end{array}$ & $\begin{array}{c}88 \\
(91.7)\end{array}$ & $\begin{array}{c}68 \\
(98.6)\end{array}$ \\
\hline $\begin{array}{l}\text { Abnormalities in the long } \\
\text { bones of the extrimities }(\%)\end{array}$ & $\begin{array}{c}9 \\
(1.6)\end{array}$ & 0 & 0 & $\begin{array}{c}28 \\
(18.8)\end{array}$ & $\begin{array}{c}48 \\
(40.7)\end{array}$ & $\begin{array}{c}57 \\
(68.7)\end{array}$ & $\begin{array}{c}87 \\
(94.6)\end{array}$ & $\begin{array}{l}100 \\
(99.1)\end{array}$ & $\begin{array}{c}88 \\
(87.5)\end{array}$ & $\begin{array}{c}68 \\
(98.6)\end{array}$ \\
\hline
\end{tabular}

Table 6 Pregnancy status of Rats treated with various doses of hypervitaminosis-A $(50,000 \sim 250,000 \mathrm{IU} / \mathrm{kg})$ on the 11 th day of pregnancy

\begin{tabular}{|c|c|c|c|c|c|c|c|c|}
\hline 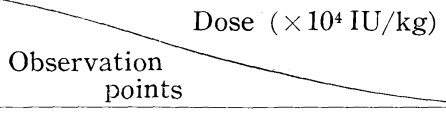 & CONT. & 5.0 & 10.0 & 15.0 & 17.5 & 20.0 & 22.5 & 25.0 \\
\hline No. of rats treated & 126 & 5 & 5 & 5 & 10 & 11 & 10 & 10 \\
\hline Total number of fetuses & 1244 & 40 & 46 & 44 & 96 & 103 & 88 & 96 \\
\hline Fetal deaths & $\begin{array}{l}48 \\
(3.9)\end{array}$ & $\begin{array}{c}2 \\
(5.0)\end{array}$ & $\begin{array}{c}3 \\
(6.5) \\
\end{array}$ & $\begin{array}{c}2 \\
(4.6) \\
\end{array}$ & $\begin{array}{c}3 \\
(3.1) \\
\end{array}$ & $\begin{array}{c}3 \\
(2.9)\end{array}$ & $\begin{array}{c}3 \\
(3.4)\end{array}$ & $\begin{array}{c}6 \\
(6.2)\end{array}$ \\
\hline Fetuses examined & $\begin{array}{l}1196 \\
1167\end{array}$ & $\begin{array}{l}38 \\
38\end{array}$ & $\begin{array}{l}43 \\
43\end{array}$ & $\begin{array}{l}42 \\
42\end{array}$ & $\begin{array}{l}93 \\
92\end{array}$ & $\begin{array}{l}100 \\
100\end{array}$ & $\begin{array}{l}85 \\
85 \\
\end{array}$ & $\begin{array}{l}90 \\
88 \\
\end{array}$ \\
\hline $\begin{array}{l}\text { No. of fetuses with gross } \\
\text { malformations }\end{array}$ & 0 & 0 & 0 & 0 & $\begin{array}{c}5 \\
(5.4)\end{array}$ & $\begin{array}{c}14 \\
(14.0)\end{array}$ & $\begin{array}{c}20 \\
(23.5)\end{array}$ & $\begin{array}{c}51 \\
(56.7)\end{array}$ \\
\hline Limb malformations $\quad(\%)$ & 0 & 0 & 0 & 0 & 0 & 0 & 0 & $\begin{array}{c}40 \\
(44.4)\end{array}$ \\
\hline Digital malformations $\quad(\%)$ & 0 & 0 & 0 & 0 & 0 & $\begin{array}{c}10 \\
(10.0)\end{array}$ & $\begin{array}{c}12 \\
(14.1)\end{array}$ & $\begin{array}{c}37 \\
(41.1)\end{array}$ \\
\hline $\begin{array}{l}\text { No. of fetuses with bone } \\
\text { abnormalities }\end{array}$ & $\begin{array}{l}17 \\
(1.5)\end{array}$ & 0 & $\begin{array}{c}3 \\
(7.0)\end{array}$ & $\begin{array}{c}4 \\
(9.5) \\
\end{array}$ & $\begin{array}{c}30 \\
(32.6) \\
\end{array}$ & $\begin{array}{c}27 \\
(27.0) \\
\end{array}$ & $\begin{array}{c}35 \\
(41.2)\end{array}$ & $\begin{array}{c}70 \\
(79.5)\end{array}$ \\
\hline $\begin{array}{l}\text { Abnormalities in the long } \\
\text { bones of the extrimities }(\%)\end{array}$ & 0 & 0 & 0 & 0 & $\begin{array}{c}4 \\
(4.4) \\
\end{array}$ & $\begin{array}{c}23 \\
(23.0) \\
\end{array}$ & $\begin{array}{c}27 \\
(31.8)\end{array}$ & $\begin{array}{c}56 \\
(63.6) \\
\end{array}$ \\
\hline
\end{tabular}

shown in Tables 5-7 and Text-figure 2A-B.

In the species employed, no clear dose-response was found in embryonic and fetal deaths or in development retarded fetuses. However, the incidence of micromelia manifested in mice and rats was elevated with increase in dosage. In this case, the incidence in each corresponding dose was always higher in mice than in rats. In order to compare difference in the threshold of teratogenic dose of vitamin A among species the minimal dose which produced $10 \%$ malformations was tentatively designated 
Table 7 Pregnancy status of rabbits treated with various doses of hypervitaminosis-A $(50,000 \sim 250,000 \mathrm{IU} / \mathrm{kg})$ on the 10 th day of pregnancy

\begin{tabular}{|c|c|c|c|c|c|c|c|c|}
\hline 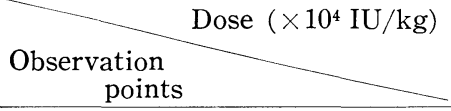 & CONT. & 5.0 & 10.0 & 15.0 & 17.5 & 20.0 & 22.5 & 25.0 \\
\hline No. of rabbits treated & 4 & 3 & 3 & 3 & 3 & 4 & 4 & 3 \\
\hline Total number of fetuses & 31 & 26 & 28 & 25 & 31 & 41 & 39 & 25 \\
\hline Fetal deaths & $\begin{array}{c}1 \\
(3.2)\end{array}$ & $\begin{array}{c}4 \\
(15.4)\end{array}$ & $\begin{array}{c}5 \\
(18.5)\end{array}$ & $\begin{array}{c}5 \\
(20.0)\end{array}$ & $\begin{array}{c}4 \\
(12.9)\end{array}$ & $\begin{array}{c}8 \\
(19.5)\end{array}$ & $\begin{array}{c}15 \\
(38.4)\end{array}$ & $\begin{array}{c}10 \\
(40.3)\end{array}$ \\
\hline Fetuses examined & $\begin{array}{l}30 \\
30\end{array}$ & $\begin{array}{l}22 \\
22\end{array}$ & $\begin{array}{l}23 \\
23\end{array}$ & $\begin{array}{l}20 \\
20\end{array}$ & $\begin{array}{l}27 \\
27\end{array}$ & $\begin{array}{l}33 \\
31\end{array}$ & $\begin{array}{l}24 \\
24\end{array}$ & $\begin{array}{l}15 \\
15 \\
\end{array}$ \\
\hline $\begin{array}{l}\text { No. of fetuses with gross } \\
\text { malformations }\end{array}$ & 0 & 0 & $\begin{array}{c}1 \\
(4.3)\end{array}$ & 0 & 0 & $\begin{array}{c}8 \\
(24.2) \\
\end{array}$ & $\begin{array}{c}16 \\
(66.7)\end{array}$ & $\begin{array}{c}5 \\
(33.3)\end{array}$ \\
\hline Limb malformations $\quad(\%)$ & 0 & 0 & $\begin{array}{c}1 \\
(4.3)\end{array}$ & 0 & 0 & 0 & 0 & 0 \\
\hline Digital malformations & 0 & 0 & $\begin{array}{c}1 \\
(4.3)\end{array}$ & 0 & 0 & $\begin{array}{c}1 \\
(3.0)\end{array}$ & $\begin{array}{c}2 \\
(8.3)\end{array}$ & $\begin{array}{c}1 \\
(6.7) \\
\end{array}$ \\
\hline $\begin{array}{l}\text { No. of fetuses with bone } \\
\text { abnormalities }\end{array}$ & $\begin{array}{c}1 \\
(3.3) \\
\end{array}$ & 0 & $\begin{array}{c}1 \\
(4.3)\end{array}$ & 0 & 0 & $\begin{array}{c}11 \\
(35.5)\end{array}$ & $\begin{array}{c}16 \\
(66.7) \\
\end{array}$ & $\begin{array}{c}6 \\
(40.0) \\
\end{array}$ \\
\hline $\begin{array}{l}\text { Abnormalities in the long } \\
\text { bones of the extrimities }\end{array}$ & 0 & 0 & $\begin{array}{c}1 \\
(4.3)\end{array}$ & 0 & 0 & $\begin{array}{c}3 \\
(9.7) \\
\end{array}$ & $\begin{array}{c}3 \\
(12.5) \\
\end{array}$ & $\begin{array}{c}3 \\
(20.0)\end{array}$ \\
\hline
\end{tabular}

as the minimal teratogenic dose. The dose for the production of micromelia was at a dose level between 100,000 IU/kg and 125,000 IU/kg in mice and between 225,000 IU/kg and $250,000 \mathrm{IU} / \mathrm{kg}$ in rats. (Text-fig. 2A) While, minimal teratogenic dose for the long bones of the extremities seems to be smaller than that for micromelia in gross observation, i.e. the minimal teratogenic dose in this case was at a dose level between $75,000 \mathrm{IU} / \mathrm{kg}$ and $100,000 \mathrm{IU} / \mathrm{kg}$ in mice and $175,000 \mathrm{IU} / \mathrm{kg}$ and $200,000 \mathrm{IU} / \mathrm{kg}$ in rats (Text-fig. 2C). No micromelia was found in rabbits at any dose levels between 50,000 $\mathrm{IU} / \mathrm{kg}$ and 250,000 IU/kg except for minor defects such as thickening of the long bones of the extremities. In such a case, the minimal effective dose was at a dose level between 200,000 IU/kg and 225,000 IU/kg.

Digital malformations were observed in all species employed and such anomalies manifested a dose-response relationship. The minimal teratogenic dose was shown to be at a dose level between $125,000 \mathrm{IU} / \mathrm{kg}$ and $150,000 \mathrm{IU} / \mathrm{kg}$ in mice, between 200,000 $\mathrm{IU} / \mathrm{kg}$ and $225,000 \mathrm{IU} / \mathrm{kg}$ in rats and between $250,000 \mathrm{IU} / \mathrm{kg}$ and $300,000 \mathrm{IU} / \mathrm{kg}$ in rabbits. (Text-fig. 2B)

\section{DISCUSSION}

It was clearly demonstrated in the three species employed that the frequency of fetal deaths, including embryonic losses, as well as of limb and digital malformations was dependent upon the developmental stage at which the excess vitamin A was administered. The earlier the stage, the higher was frequency of fetal deaths including embryonic losses in all the species used. When frequency is compared at each equivalent age of the embryos in the three species, the frequency was the highest in rabbits, 

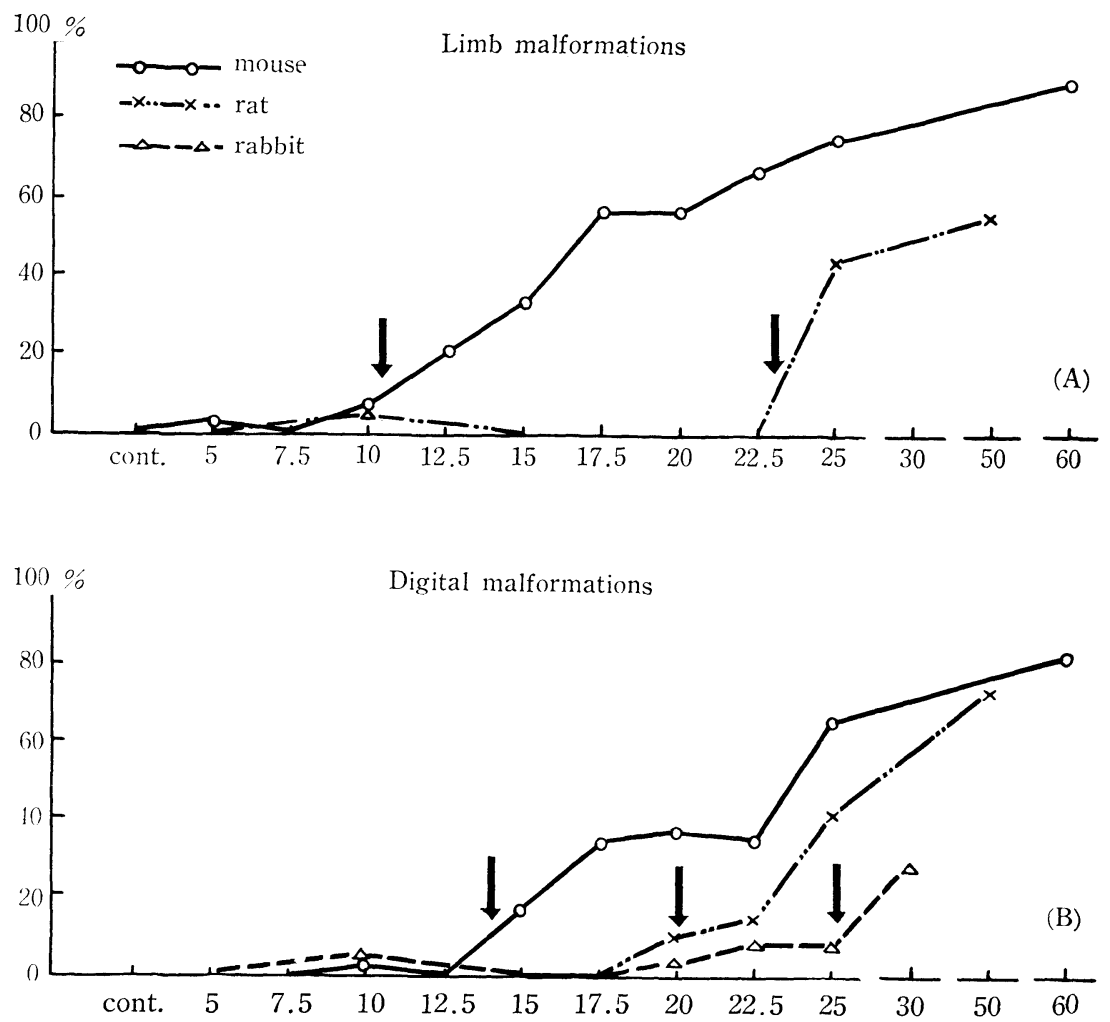

Malformation of the long bones of the extremities

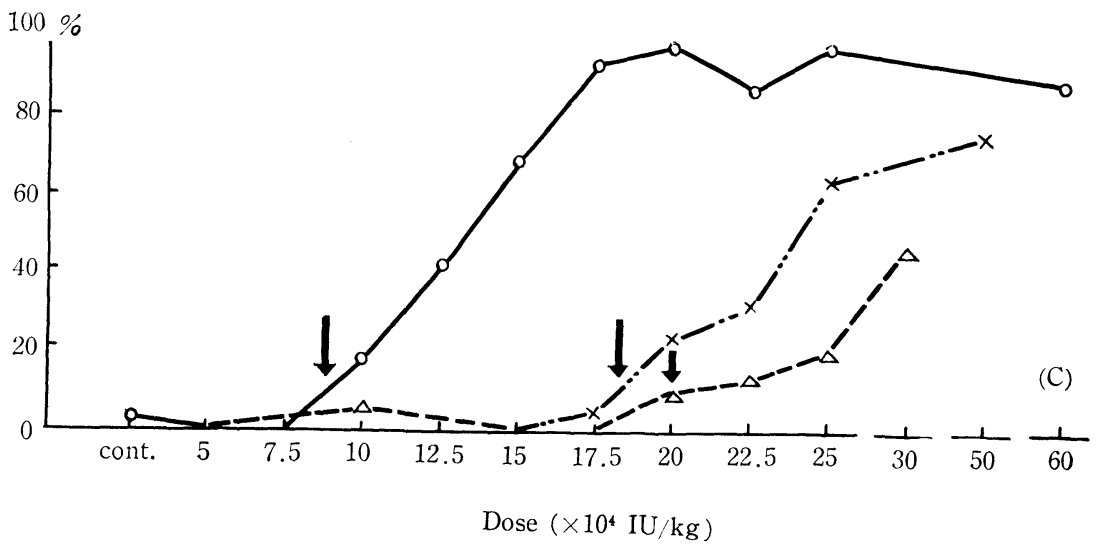

Text-fig. 2. Relationships between various doses of vitamin $A$ and the incidence of limb and digital malformations. Arrow indicates minimal teratogenic dose. A, limb malformations. B, digital malformations. $C$, malformations of the long bones of the extremities.

next in mice and the lowest in rats.

The most striking feature of the present experiments was the greatly increased incidence of micromelia and ectrodactylia in mice and rats in comparison with the 
absence of micromelia as well as the low frequency of ectrodactylia in rabbits. However, without further study, the likelihood that the increased mortality of embryos in rabbits is unrelated to the absence of micromelia and the low frequency of ectrodactylia in this species should not be ruled out. The critical stage for the occurrence of micromelia and digital malformations in mice was one to two days earlier compared with that in rats. The developmental stage in mice also was approximately two days earlier in comparison with the equivalent stage in rats. The susceptible period for the production of digital malformations of the forelimb in rabbits was the 10th to the 13th day of pregnancy. According to Text-figure 1 such a period in the rabbit corresponds to the 10th to the 11th day in the mouse and the 11 th to the $131 / 2$ th day in the rat, and these periods in mice and rats are included in the susceptible period for the production of digital malformations in both species. Therefore, it is evident that the difference among species in the critical stage for the occurrence of limb and digital malformations is related to the difference in the developmental stage. In other words, the difference among species in the critical stage for such malformations corresponds to the difference among species in the time of formation as well as differentiation of limb buds and hand or foot plates. The difference of the critical stage between the forelimb and hindlimb may also be due to a difference in the time of formation as well as differentiation of limb buds in the fore- and hindlimbs.

There was no difference between mice and rats in the nature of the skeletal system in micromelia. The micromelia was precisely similar to that described by Kalter and Warkany (1961), Murakami et al. (1963, 1965), Kameyama et al. (1964) and Esaki (1964), as occurring in the fetuses of female subjected to hypervitaminosis A during pregnancy. Similar deformities have also been reported by Giroud and Martinet (1956) and Woollam and Millen (1963). The occurrence of digital malformations has been described in the references described above.

The dose-response relationship in the production of limb and digital malformations and the minimal teratogenic dose, which was determined by dose-response curve for each anomaly under study, were shown to vary greatly according to species employed. Embryonic susceptibility to the teratogenic action of hypervitaminosis A, which was estimated from the dose-response curve and minimal teratogenic dose, was the highest in mice, next in rats and the lowest in rabbits. Similar results have been suggested by a brief report made by Giroud and Martinet (1958). According to these investigators, the teratogenic action of hypervitaminosis $\mathrm{A}$, which was injected at a comparatively earlier stage of development, was more prominent in mice and rats than in rabbits and guinea pigs.

In spite of numerous experiments, the mechanism of teratogenic effect of hypervitaminosis $\mathrm{A}$ as well as the normal action of vitamin A except for a role of vitamin A on the retina are still obscure. Murakami et al. (1965) have stated that hypervitaminosis A exerted a suppressive effect chiefly upon the mesenchymal tissues just before 
their differentiation into primodium or on the mesenchymal tissues duriug differentiation. A study of the difference among species in normal role of vitamin A may be a prerequisite to study on difference among species in teratogenic action as well as teratogenic mechanism of hypervitaminosis A.

Generally, it was found in the present investigation that embryonic susceptibility expressed by the incidence of limb and digital malformations was the highest in the mouse, next in the rat and the lowest in the rabbit. However, such a difference among species in embryonic susceptibility to hypervitaminosis A may be not absolute, because 1) equivalent ages were determined only by gross observation of several morphogenetic patterns, 2) minimal teratogenic dose is subjected to the strain used and the method of analysis, and 3) teratogenic action of extrinsic agents might be related to the developmental potency of embryos. From these viewpoints, it is desirable to conduct a more detailed and reliable comparison of developmental stage in each species employed. These studies are in progress.

\section{SUMMARY}

The present paper deals with a) a gross comparison of the embryonic stage in CF-1 mice, Sprague Dawley rats and albino rabbits, b) the relationship between the stage at which hypervitaminosis $\mathrm{A}$ was administered and the incidence of fetal deaths including embryonic losses as well as of limb and digital malformations, and c) the relationship between various dose levels of hypervitaminosis $\mathrm{A}$ and malformation of the extremities in the three species.

Generally, susceptibility expressed by fetal deaths, including embryonic losses, seems to be the highest in the rabbit, next in the mouse, and the lowest in the rat, while susceptibility of limb buds and hand or foot plates to the teratogenic action of hypervitaminosis A seems to be the highest in the mouse, next in the rat and the lowest in the rabbit.

\section{ACKNOWLEDGEMENTS}

The authors wish to express their gratitude to Professor U. Murakami, Research Institute of Environmental Medicine, Nagoya University, for his valuable suggestions and criticism in carrying out the present study.

Further thanks are given to Dr. S. Tanaka for his constructive advice and friendly assistance and to Miss K. Ōta and Messers. H. Kanamori, M. Tada and O. Takatani for their skillful technical assistance. 


\section{LITERATURE CITED}

Baba, T., and E. Araki, 1959 Morphogenesis of malformation due to excessive vitamin A (1). Osaka City Med. J. 5: 9-15,

Cohlan, S. Q., 1953 Excessive intake of vitamin A as a cause of congenital anomalies in the rat. Science 117: 535-536.

Dawson, A. B., 1926 A note on the staining of the skeleton on cleared specimens with alizarin red S. Stain Technol. 1: 123-124.

Deuschle, F. M., J.F. Geiger, and J. Warkany, 1959 Analysis of anomalous oculodentofacial pattern in newborn rats produced by maternal hypervitaminos A. J. Dental Res. 38: 149-155.

Esaki, K., 1964 Effects of treatment with hypervitaminosis A in pregnant mice. 3. External malformations of live fetuses (II). (in Japanese) Cong. Anom. (Official J. Cong. Anom. Res. Assoc. Jap.) 4 : 43-51.

Fraser, F.C., 1963 Experimental teratogenesis in relation to congenital malformations in man. In: 2nd Intern. Conf. Con. Malf., Lippincott, Philadelphia, Montreal.

Giroud, A., and M. Martinet, 1955 Hypervitaminose A et anomalies chez le fetus de lat. Internat. Z. Vitaminoforsch. 26: 10-18.

Giroud, A., and M. Martinet, 1956 Tératogenèse par hautes doses de vitamine A en fonction des stades dévelopment. Arch. Anat. Microscop. Morphol. Exp. 45: 77-98.

Giroud, A., and M. Martinet, 1958 Tératogènese par hypervitaminose A chez le souris, le cobaye et le lapin. Arch. Franc Pediat. 16: 1-5.

Ihara, T., M. Mizutani, S. Tanaka, and K. Kaziwara, 1966 Effect of hypervitaminosis A upon the fetuses of some rodents, with special reference to oculofacial syndrome. (in Japanese) Cong. Anom. (Official J. Cong. Anom. Res. Assoc. Jap.) 6, in press.

Kalter, H., 1960 Teratogenic effects of hypervtaminosis A upon face and mouth of inbred mice. Ann. N. Y. Acad. Sci. 85: 42-55.

Kalter H., and J. Warkany, 1961 Experimetal production of congenital malformations in strains of inbred mice by maternal treatment with hypervitaminosis A. Am. J. Pathol. 38: 1-21.

Kameyama, Y., H. Nogami, and U. Murakami, 1964 Study on micromelias in the mouse fetuses caused by X-irradiation, hypoxia, trypan blue injection or hypervitaminosis A upon mother animals during pregnancy. Ann. Rept. Environ. Med. Nagoya Univ. 12: 29-41.

Millen, J.W., and D. H. Woollam, 1957 Influence of cortisone on teratogenic effects of hypervitaminosis A. Brit. Med. J. 5038, 196-197.

Millen, J.W., and D. H. Woollam, 1963 Congenital malformations of the skeletal system. Proc. Europ. Soc. Study Drug Toxicity 1: 9-24.

Murakami, U. and Kameyama, M. 1965 Malformations of the mouse fetus caused by hypervitaminosis A of the mother during pregnancy. Arch. Environ. Health 10: 732-741.

Murakami, U., Y. Kameyama, M. Mizutani, T. Ihara, K. Esaki, H. Hamanaka, M. Imai, and Y. Ito, 1963 Studies on intrauterine environment: Experimental formation of malformations in abnormal intrauterine environment. 1. Malformations of the mouse fetus caused by hypervitaminosis A of their mothers during pregnancy (Preliminary report) (in Japanese). Ann. Rept. Res. Inst. Environ. Med. Nagoya Univ. 15: 142-149.

Ohzu, E., and R. Shoji, 1965 Some aspects on the effect of vitamin A on developing mouse embryos. Proc. Japan Acad. 41: 83-85.

Takekoshi, S., 1964 The mechanism of vitamin A induced teratogenesis. J. Embryol. Exp. Morphol. 12: $263-371$.

Wilson, J. G., 1959 Experimental studies on congenital malformations. J. Chronic. Diseases 10: 111130.

Woollam, D, H., and J.W. Millen, 1961 Influence of uterine position on response of mouse embryo to tetoragenic effects of hypervitaminosis A. Nature 190: 184-185. 